\title{
On Constant Metric Dimension of Some Generalized Convex Polytopes
}

\author{
Xuewu Zuo (D), ${ }^{1}$ Abid Ali $\left(D,{ }^{2}\right.$ Gohar Ali $\left(\mathbb{D},{ }^{2}\right.$ Muhammad Kamran Siddiqui $\left(\mathbb{D},{ }^{3}\right.$ \\ Muhammad Tariq Rahim (10, ${ }^{4}$ and Anton Asare-Tuah $\mathbb{1}^{5}$ \\ ${ }^{1}$ Department of General Education, Anhui Xinhua University, Hefei, China \\ ${ }^{2}$ Department of Mathematics, Islamia College, Peshawar, Khyber Pakhtunkhwa, Pakistan \\ ${ }^{3}$ Department of Mathematics, COMSATS University Islamabad, Lahore Campus, Pakistan \\ ${ }^{4}$ Department of Mathematics, Abbottabad University of Science and Technology, Abbottabad, Khyber Pakhtunkhwa, Pakistan \\ ${ }^{5}$ Department of Mathematics, University of Ghana, Legon, Ghana
}

Correspondence should be addressed to Anton Asare-Tuah; aasare-tuah@ug.edu.gh

Received 12 June 2021; Accepted 31 July 2021; Published 10 August 2021

Academic Editor: Antonio Di Crescenzo

Copyright ( $\odot 2021$ Xuewu Zuo et al. This is an open access article distributed under the Creative Commons Attribution License, which permits unrestricted use, distribution, and reproduction in any medium, provided the original work is properly cited.

\begin{abstract}
Metric dimension is the extraction of the affine dimension (obtained from Euclidean space $E^{d}$ ) to the arbitrary metric space. A family $\mathscr{F}=\left(G_{n}\right)$ of connected graphs with $n \geq 3$ is a family of constant metric dimension if $\operatorname{dim}(G)=k$ (some constant) for all graphs in the family. Family $\mathscr{F}$ has bounded metric dimension if $\operatorname{dim}\left(G_{n}\right) \leq M$, for all graphs in $\mathscr{F}$. Metric dimension is used to locate the position in the Global Positioning System (GPS), optimization, network theory, and image processing. It is also used for the location of hospitals and other places in big cities to trace these places. In this paper, we analyzed the features and metric dimension of generalized convex polytopes and showed that this family belongs to the family of bounded metric dimension.
\end{abstract}

\section{Introduction}

Let $G \in \mathscr{F}$ be a finite, simple, and undirected connected graph with vertex set $V=V(G)=\left\{v_{1}, v_{2}, \ldots, v_{n}\right\}$ and edge set $E=E(G)$. The distance between two vertices is denoted by $\mathrm{d}\left(v_{s}, v_{j}\right)=d_{s j}$ where $d_{s j}$ is the length of the shortest path between these vertices in $G$. Moreover, the distance $d_{s j}=d_{j s}$ because all graphs are undirected. An ordered subset $W=$ $\left\{w_{1}, w_{2}, \ldots, w_{k}\right\}$ of $V$ is called a resolving set or locating set for $G$ if for any two distinct vertices $v_{s}$ and $v_{j}$, their codes are distinct with respect to $Z$, where $\operatorname{code}\left(v_{s}\right)=$ $\left(\mathrm{d}\left(v_{s}, z_{1}\right), \mathrm{d}\left(v_{s}, z_{2}\right), \ldots, \mathrm{d}\left(v_{s}, z_{k}\right)\right) \in \mathbb{W}^{k}$ is a vector [1]. $\min :\{|W|: W$ is a resolving set of $G\}=\operatorname{dim}(G)=\beta(G)$ is called the metric dimension or locating number of $G$, and such a resolving set $Z$ is called a basis set for $G$. To investigate $Z$ is a basis set for $G$, it suffices to show that, for all different vertices $x, y \in V \backslash W$, their codes are also different because for any $w_{j} \in W, 1 \leq j \leq k$, the $j$ th component of the code is zero, while all other components are nonzero. For more details about $\beta(G)$ and resolving sets, one can read [1-4].
Lemma 1 (see [3]). For a connected graph $G$ with resolving set $W$, if $d\left(x_{s}, w\right)=d\left(x_{j}, w\right)$ for all $w \in V \backslash\left\{x_{s}, x_{j}\right\}$, then $W \cap\left\{x_{s}, x_{j}\right\} \neq \varnothing$.

The join of two graphs $G$ and $H$ represented as $G+H$ is a graph with $V(G+H)=V(G) \cup V(H)$ and $E(G+H)=E(G) \cup E(H) \cup\{g h: g \in V(G)$ and $h \in V(H)\}$. $W_{n}=C_{n}+K_{1}$ is a wheel graph of order $n+1$ for $n \geq 3$. $f_{n}=$ $P_{n}+K_{1}$ is a fan graph obtained from the amalgamation of the path on $n$ vertices with a single vertex graph $K_{n}$. Jahangir or gear graph $J_{2 n}$ is obtained from the wheel graph $W_{2 n}$ by deleting $n$-cycle edges alternatively; see in [4]. The following results appear in [5-7] for the graphs defined above.

Theorem 1. For wheel graph $W_{n}$, fan graph $f_{n}$, and Jahangir graph $J_{2 n}$, we have the following:

(i) $\beta\left(W_{n}\right)=\lfloor(2 n+2) / 5\rfloor$, for every $n \geq 7$

(ii) $\beta\left(f_{n}\right)=[(2 n+2) / 5]$, for every $n \geq 7$ 
(iii) $\beta\left(J_{2 n}\right)=\lfloor 2 n / 3\rfloor$, for every $n \geq 4$

All the above three families of graphs are planar, and their metric dimension depends on the number of vertices in the graph, which shows that the metric dimension of these graphs is unbounded [8,9]. Khuller et al. [10] clarified the properties of those graphs whose metric dimension is two.

Theorem 2 (see [10]). Let $\beta(G)=2=|W|$, where $W=\{x, y\} \subset V(G)$; then, the following holds:

(i) There is a unique shortest path $P$ between $x$ and $y$

(ii) $\operatorname{deg}(x) \leq 3$ or $\operatorname{deg}(y) \leq 3$

(iii) For every other vertex $z$ except $x$ and $y$ on $P$, $\operatorname{deg}(z) \leq 5$

Definition 1 (see [11]). A set $K \subset \mathbb{R}^{d}$ is said to be convex if the line segment $\overline{x y}: \lambda x+(1-\lambda) y, 0 \leq \lambda \leq 1$, lies inside $K$ for all distinct pairs of point $x, y \in K$.

Definition 2 (see [11]). The smallest convex set containing $K$ (the intersection of the family of all convex sets that contain $K)$ is called the convex hull of $K$, denoted by $\operatorname{Conv}(K)=\cap_{K \subset S_{s}} S_{s}$, where $S_{s}$ is a convex set.

Definition 3 (see [11]). A convex polytope is a bounded convex linear combination of convex sets.

There are some families of graphs with constant metric dimension (see [2]); these families are generated by convex polytopes. The problem of finding $\beta(G)$ is NP-complete (see [2]).

Theorem 3 (see [12]). Let $S_{n}^{p}$ be a convex polytope with p-pendent vertices; then, $\operatorname{dim}\left(S_{n}^{p}\right)=3$ for all $n \geq 6$.

Theorem 4 (see [12]). The metric dimension of convex polytope $T_{n}^{p}$ with $p$-pendent edges is 3 for every $n \geq 6$.

Theorem 5 (see [12]). $\beta\left(U_{n}^{p}\right)=3$ for $n \geq 6$, where $U_{n}^{p}$ is a convex polytope graph with p-pendents.

For more details about the metric dimension of certain families of graphs, see $[13,14]$. Here, we will investigate generalized convex polytopes with pendent edges for their metric dimensions.

\section{Main Results}

This section is devoted to the main results which we proved for the newly introduced generalized convex polytopes. The convex polytopes $S_{n}, T_{n}$, and $U_{n}$ were examined by Muhammad et al. for their metric dimensions in [2] and proved that these families belong to the family of constant metric dimension.

Generalized convex polytope $S_{n, m}$ is the generalization of $S_{n}$, with one $n$-sided and infinite face each, 3 -sided faces being $2 n$, and 4 -sided faces being $n(m-2)$, so the total number of faces is $n m+2$. The convex polytope $S_{n, m}^{p}$ is obtained from the generalized convex polytope graph by attached $p$-pendent vertices at the outer cycle of $S_{n, m}$, shown in Figure 1. The generalized convex polytope $S_{n . m}^{p}$ with $p$-pendents is a graph consisting of $m$ cycles, with vertex and edge sets

$$
\begin{aligned}
V\left(S_{n, m}^{p}\right) & =\left\{X_{s}^{j}: 1 \leq s \leq n, 1 \leq j \leq m\right\}, \\
E\left(S_{n, m}^{p}\right) & =\left\{X_{s}^{j} X_{s+1}^{j}: 1 \leq s \leq n, 1 \leq j \leq m\right\} \\
& \cup\left\{X_{s}^{j} X_{s}^{j+1}: 1 \leq s \leq n, 1 \leq j \leq m\right\} \\
& \cup\left\{X_{s+1}^{1} X_{s}^{2}: 1 \leq s \leq n\right\} .
\end{aligned}
$$

In the set of edges, indices are taken as modulo $n$ and $m$. In [2], it was shown that $\beta\left(S_{n}\right)=3$, for $n \geq 6$. In the result below, we proved that the metric dimension for the generalized convex polytope of $S_{n}$ is still 3, which implies that $S_{n}$, $S_{n}^{p}$, and $S_{n, m}^{p}$ belong to the same family of constant metric dimension.

Theorem 6. Let $G=S_{n, m}^{p}$ be the generalized convex polytope graph defined above; then, $\beta(G)=3$ for $n \geq 6$ and $m \geq 5$.

Proof. Validating the mentioned theorem with the help of double inequalities, two cases are present:

Case (i): for $n$ is even.

Let $n=2 \alpha^{\prime}$ where $\alpha^{\prime} \geq 3$ is an integer. As $\left|N_{2}(x)\right| \geq 6$ for all $x \in S_{n, m}$, it is guaranteed by [15] that $\beta(G) \geq 3$.

Consider $Z=\left\{X_{1}^{1}, X_{2}^{1}, X_{l+1}^{1}\right\}$ to be an ordered subset of $V\left(S_{n, m}^{p}\right)$; to show that $Z$ is a basis set for $G$, codes of the elements of $V\left(S_{n, m}^{p}\right) \backslash Z$ with respect to $Z$ are given in the following scheme:

$$
\begin{aligned}
& r\left(X_{s}^{1} \mid Z\right)= \begin{cases}\left(s-1, s-2, \alpha^{\prime}-s+1\right), & \alpha^{\prime} \geq s \geq 3, \\
\left(2 \alpha^{\prime}-s+1,2 \alpha^{\prime}-s+2, s-\alpha^{\prime}-1\right), & 2 \alpha^{\prime} \geq s \geq \alpha^{\prime}+2,\end{cases} \\
& r\left(X_{s}^{2} \mid Z\right)= \begin{cases}\left(1,1, \alpha^{\prime}\right), & s=1, \\
\left(s, s-\alpha^{\prime}, \alpha^{\prime}-s+1\right), & \alpha^{\prime} \geq s \geq 2, \\
\left(\alpha^{\prime}, \alpha^{\prime}, 1\right), & s=\alpha^{\prime}+1, \\
\left(2 \alpha^{\prime}-s+1,2 \alpha^{\prime}-s+2, s-l\right), & 2 \alpha^{\prime} \geq s \geq \alpha^{\prime}+2 .\end{cases}
\end{aligned}
$$




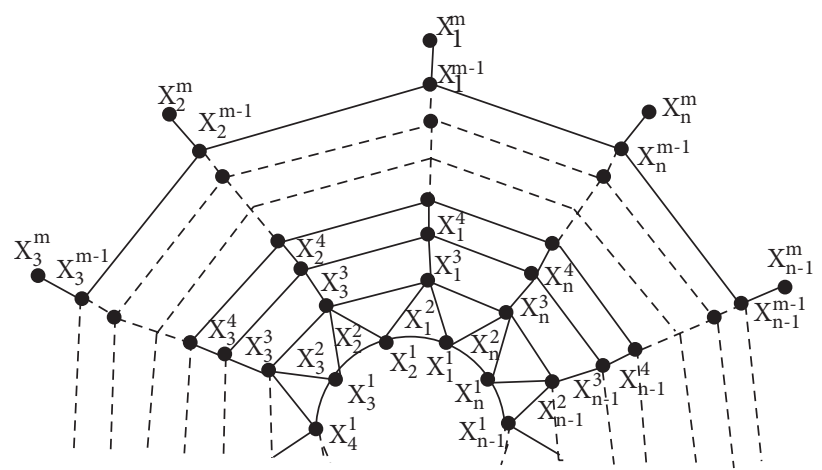

FIgURE 1: The generalized convex polytope graph $S_{n, m}^{p}$.

Codes for the vertices $X_{s}^{m}$ for $1 \leq s \leq n$ and $m \geq 3$ are given in the following:

$$
r\left(X_{s}^{m} \mid Z\right)=(m-2, m-2, m-2)+r\left(X_{s}^{2} \mid Z\right) .
$$

It proves that $\beta(G) \leq 3$ implies that the metric dimension of $G=S_{n, m}^{p}$ is 3 .

Case (ii): for $n$ is an odd integer.
Let $n=2 \alpha^{\prime}+1$, where $\alpha^{\prime} \geq 3$, and by [15], $\beta(G) \geq 3$; for reaching the conclusion, it remains to show that $\beta(G) \leq 3$.

Let $Z=\left\{X_{1}^{1}, X_{2}^{1}, X_{l+1}^{1}\right\}$ be an ordered subset of $S_{n, m}^{p}$; the formulation for the representation of nodes for $V\left(S_{n, m}^{p}\right) \backslash Z$ with respect to $Z$ is given in the following:

$$
\begin{aligned}
& r\left(X_{s}^{1} \mid Z\right)= \begin{cases}\left(s-1, s-2, \alpha^{\prime}-s+1\right), & \alpha^{\prime} \geq s \geq 3, \\
\left(\alpha^{\prime}, \alpha^{\prime}, 1\right), & s=\alpha^{\prime}+2, \\
\left(2 \alpha^{\prime}-s+2,2 \alpha^{\prime}-s+3, s-\alpha^{\prime}-1\right), & 2 \alpha^{\prime}+1 \geq s \geq \alpha^{\prime}+3,\end{cases} \\
& r\left(X_{s}^{2} \mid Z\right)= \begin{cases}\left(1,1, \alpha^{\prime}\right), & s=1, \\
\left(s, s-\alpha^{\prime}, \alpha^{\prime}-s, l-s+1\right), & \alpha^{\prime} \geq s \geq 2, \\
\left(\alpha^{\prime}+1, \alpha^{\prime}, 1\right), & s=\alpha^{\prime}+1, \\
\left(2 \alpha^{\prime}-s+2,2 \alpha^{\prime}-s+3, s-\alpha^{\prime}\right), & n \geq s \geq \alpha^{\prime}+2 .\end{cases}
\end{aligned}
$$

The representation of the vertices $X_{s}^{m}, 1 \leq s \leq n$ and $m \geq 3$, is as follows:

$$
r\left(X_{s}^{m} \mid Z\right)=(m-2, m-2, m-2)+r\left(X_{s}^{2} \mid Z\right) .
$$

It shows that, for any two distinct vertices $x, y \in S_{n, m}^{p}$ for odd $n \geq 7, r(x \mid Z) \neq r(y \mid Z)$ implying that $\beta(G) \leq 3$; this completes the proof.

\section{Generalized Convex Polytope Graph $T_{n, m}^{p}$}

In [16], Imran et al. proved the metric dimension of convex polytope $T_{n}$. The general form of $T_{n}$ is denoted by $T_{n, m}$ known as the generalized convex polytope (for short, GCP); this graph consists of one each $n$-sided and infinite face, respectively, and the number of 3 -sided faces is $4 n$ and 4sided faces is $n(m-3)$. The GCP graph $T_{n, m}^{p}$ is a graph with $p$-pendent edges. Vertex and edge sets for $G=T_{n, m}^{p}$ are given in the following:

$$
\begin{aligned}
V\left(T_{n, m}^{p}\right) & =\left\{X_{s}^{j}: 1 \leq s \leq n, 1 \leq j \leq m\right\}, \\
E\left(T_{n, m}^{p}\right) & =\left\{X_{s}^{j} X_{s+1}^{j}: 1 \leq s \leq n, 1 \leq j \leq m\right\} \\
& \cup\left\{X_{s}^{j} X_{s}^{j+1}: 1 \leq s \leq n, 1 \leq j \leq m-3\right\} \\
& \cup\left\{X_{s+1}^{m-2} X_{s}^{m-1}: 1 \leq s \leq n\right\} \cup\left\{X_{s}^{m-1} X_{s}^{m}: 1 \leq s \leq n\right\} .
\end{aligned}
$$

In the set of edges, indices are taken as modulo $n$ and $m$. In Figure 2, the graph $G=T_{n, m}^{p}$ is shown.

The result given below shows that $T_{n, m}^{p}$ belongs to the family of constant metric dimension.

Theorem 7. Let $T_{n, m}^{p}$ be a GCP graph with p-pendents for all $n \geq 6$; then, $\beta\left(T_{n, m}^{p}\right)=3$.

Proof. As $\left|N_{2}(v)\right| \geq 6$ for all nonpendent vertices of $T_{n, m}^{p}$, $\beta\left(T_{n, m}^{p}\right) \geq 3$ for all $n \geq 6$ by [15]. To complete the proof, it suffices to show that any ordered subset of the vertices of this graph is a resolving set. 
Case (i): for $n$ is an even integer.

Let $n=2 \alpha^{\prime}$ with $\alpha^{\prime} \geq 3$; consider an ordered subset $Z=$ $\left\{X_{1}^{1}, X_{2}^{1}, X_{l+1}^{1}\right\}$ of vertices of $T_{n, m}^{p}$. The representation of vertices of $V\left(T_{n, m}^{p}\right) \backslash Z$ with respect to $Z$ is formulated as follows:

$$
\begin{aligned}
& r\left(X_{s}^{1} \mid Z\right)= \begin{cases}\left(s-1, s-2, \alpha^{\prime}-s+1\right), & \alpha^{\prime} \geq s \geq 3, \\
\left(2 \alpha^{\prime}-s+\alpha^{\prime}, 2 \alpha^{\prime}-s+2, s-\alpha^{\prime}-1\right), & 2 \alpha^{\prime} \geq s \geq \alpha^{\prime}+2,\end{cases} \\
& r\left(X_{s}^{2} \mid Z\right)= \begin{cases}\left(1,1, \alpha^{\prime}\right), & s=1, \\
\left(s, s-\alpha^{\prime}, \alpha^{\prime}-s+1\right), & \alpha^{\prime} \geq s \geq 2, \\
\left(\alpha^{\prime}, \alpha^{\prime}, 1\right), & s=\alpha^{\prime}+1, \\
\left(2 \alpha^{\prime}-s+1,2 \alpha^{\prime}-s+2, s-\alpha^{\prime}\right), & 2 \geq s \geq \alpha^{\prime}+2 .\end{cases}
\end{aligned}
$$

For $3 \leq j \leq m-2$,

$$
\begin{aligned}
r\left(X_{s}^{j} \mid Z\right) & =(j-2, j-2, j-2)+s\left(X_{s}^{2} \mid Z\right) . \\
r\left(X_{s}^{m-1} \mid Z\right) & \begin{cases}\left(2+1,2+1, \alpha^{\prime}+1\right), & s=1, \\
\left(s+2, s+1, \alpha^{\prime}-s+2\right), & \alpha^{\prime}-1 \geq s \geq 2, \\
\left(\alpha^{\prime}+2, \alpha^{\prime}+1,3\right), & s=\alpha^{\prime}, \\
\left(\alpha^{\prime}+1, \alpha^{\prime}+2,3\right), & s=\alpha^{\prime}+1, \\
\left(2 \alpha^{\prime}-s+2,2 \alpha^{\prime}-s+3, s-\alpha^{\prime}+2\right), & \\
\alpha^{\prime}+2 \leq s \leq n-1, & s=n . \\
\left(3,3, \alpha^{\prime}+2\right), & \end{cases}
\end{aligned}
$$

Codes of the pendent vertices are given as follows:

$$
r\left(X_{s}^{m} \mid Z\right)=(1,1,1)+r\left(X_{s}^{m-1} \mid Z\right) .
$$

From the above formulation, it is obvious that no two distinct vertices of the GCP with pendents $p$ have the same code with respect to $Z$, which implies that $\beta\left(T_{n, m}^{p}\right)=3$.
Case (ii): for $n$ is an odd integer.

Let $n=2 \alpha^{\prime}+1$ for $\alpha^{\prime} \geq 3$; suppose an ordered subset $Z=\left\{x_{1}^{1}, X_{2}^{1}, X_{l+1}^{1}\right\}$ of vertices $V\left(T_{n, m}^{p}\right)$; to show that $Z$ is a basis set for $T_{n, m}^{p}$, the formulation codes are given as follows:

$$
\begin{aligned}
& r\left(X_{s}^{1} \mid Z\right)= \begin{cases}\left(s-1, s-2, \alpha^{\prime}-s+1\right), & \alpha^{\prime} \geq s \geq 3, \\
\left(\alpha^{\prime}, \alpha^{\prime}, 1\right), & s=\alpha^{\prime}+2, \\
\left(2 \alpha^{\prime}-s+2,2 \alpha^{\prime}-s+3, s-\alpha^{\prime}-1\right), & n \geq s \geq \alpha^{\prime}+3,\end{cases} \\
& r\left(X_{s}^{2} \mid Z\right)= \begin{cases}\left(1,1, \alpha^{\prime}\right), & s=1, \\
\left(s, s-1, \alpha^{\prime}-s+1\right), & \alpha^{\prime} \geq s \geq 2, \\
\left(\alpha^{\prime}+1, \alpha^{\prime}, 1\right), & s=\alpha^{\prime}+1, \\
\left(2 \alpha^{\prime}-s+2,2 \alpha^{\prime}-3, s-\alpha^{\prime}-1\right), & n \geq s \geq \alpha^{\prime}+2 .\end{cases}
\end{aligned}
$$


Codes given to the vertices of other interior cycles are

$$
r\left(X_{s}^{j} \mid Z\right)=(j-2, j-2, j-2)+r\left(X_{s}^{2} \mid Z\right), \quad \text { for } 1 \leq j \leq m-2 .
$$

Representation given to the second last cycle $X_{s}^{m-1}$ is

$$
r\left(X_{s}^{m-1} \mid Z\right)= \begin{cases}\left(2+1,2+1, \alpha^{\prime}+1\right), & s=1, \\ \left(s+2, s+1, \alpha^{\prime}-s+2\right), & \alpha^{\prime}-1 \geq s \geq 2, \\ \left(\alpha^{\prime}+2, \alpha^{\prime}+1,3\right), & s=\alpha \prime, \\ \left(\alpha^{\prime}+2, \alpha^{\prime}+2,3\right), & s=\alpha^{\prime}+1, \\ \left(2 \alpha^{\prime}-s+3,2 \alpha^{\prime}-s+4, s-\alpha^{\prime}+2\right), & \\ \left(n-1 \geq s \geq \alpha^{\prime}+2\right), & s=n . \\ \left(3,3, \alpha^{\prime}+2\right), & \end{cases}
$$

The same representation is given to the pendent vertices:

$$
r\left(X_{s}^{m} \mid Z\right)=(1,1,1)+r\left(X_{s}^{m-1} \mid Z\right) .
$$

It shows that $Z$ is a resolving set for $T_{n, m}^{p}$ for $n$-odd and $p$-pendents, $\beta\left(T_{n, m}^{p}\right)=3$, and this completes the proof.

\section{Generalized Convex Polytope Graph $U_{n, m}^{p}$}

In [2], the graph $U_{n}$ is given, and a generalized graph $U_{n, m}^{p}$ of $U_{n}$ is shown in Figure 3. The vertex and edge sets for this graph are given as follows:

$$
\begin{aligned}
V\left(U_{n, m}^{p}\right) & =\left\{X_{s}^{j}: 1 \leq s \leq n, s \leq j \leq m\right\}, \\
E\left(U_{n, m}^{p}\right) & =\left\{X_{s}^{j} X_{s+1}^{j}: 1 \leq s \leq n, 1 \leq j \leq m-1\right\} \\
& \cup\left\{X_{s}^{j} X_{s}^{j+1}: 1 \leq s \leq n, 1 \leq j \leq m-4\right\} \\
& \cup\left\{X_{s}^{m-3} X_{s}^{m-2}: 1 \leq s \leq n\right\} \\
& \cup\left\{X_{s}^{m-2} X_{s+1}^{m-3}: 1 \leq s \leq n\right\} \\
& \cup\left\{X_{s}^{m-2} X_{s}^{m-1}: 1 \leq s \leq n\right\} \\
& \cup\left\{X_{s}^{m-1} X_{s}^{m}: 1 \leq s \leq n\right\} .
\end{aligned}
$$

We will show that GCP graph $U_{n, m}^{p}$ with $n \geq 6$ along with $p$-pendent vertices belongs to the family of constant metric dimension and its locating number is 3 .

Theorem 8. Let $U_{n, m}^{p}$ be a GCP graph for $n \geq 6$; then, $\operatorname{dim}\left(U_{n, m}^{p}\right)=3$.

Proof. According to [15], $\operatorname{dim}(G) \geq 3$ if and only if $\left|N_{2}(x)\right| \geq 6$ or $\left|N_{3}(x)\right| \geq 8$ for all $x \in V(G)$ as $\left|N_{2}(x)\right| \geq 6$ for every nonpendent vertex $x$ of $U_{n, m}^{p}$ implying that $\operatorname{dim}\left(U_{n, m}^{p}\right) \geq 3$. To reach the conclusion, it remains to show that there exists a resolving set for $U_{n, m}^{p}$ with exactly three elements. For this, consider the following two cases:

Case (i): for an integer $n$ is even. Let $n=2 \alpha^{\prime}$, where $\alpha^{\prime} \geq 3$; take $Z=\left\{X_{1}^{1}, X_{2}^{1}, X_{l+1}^{1}\right\}$ to be an ordered subset of $V\left(U_{n, m}^{p}\right)$; to show that $Z$ resolves vertices of the GCP, the representation of vertices of the GCP is shown as follows:

$$
r\left(X_{s}^{1} \mid Z\right)= \begin{cases}\left(s-1, s-2, s-\alpha^{\prime}+1\right), & \alpha^{\prime} \geq s \geq 3, \\ \left(2 \alpha^{\prime}-s+1,2 \alpha^{\prime}-s+2, s-\alpha^{\prime}-1\right), & 2 \alpha^{\prime} \geq s \geq \alpha^{\prime}+2 .\end{cases}
$$




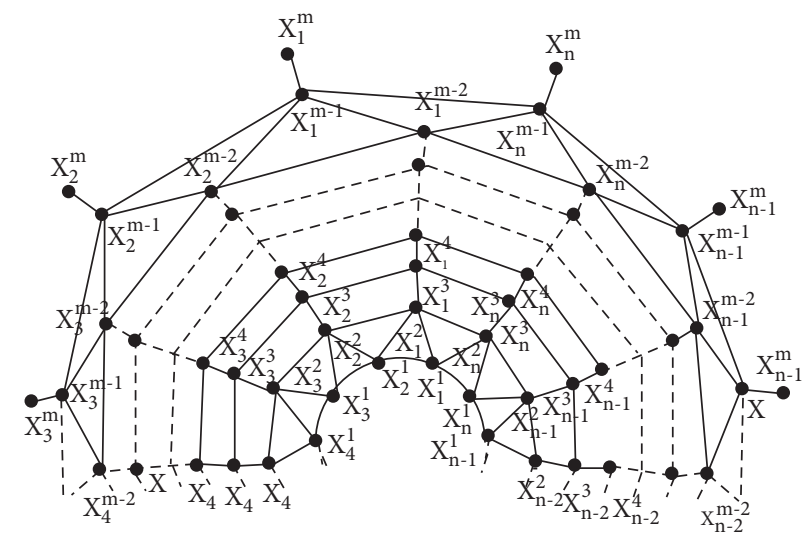

Figure 2: GCP graph $T_{n, m}^{p}$.

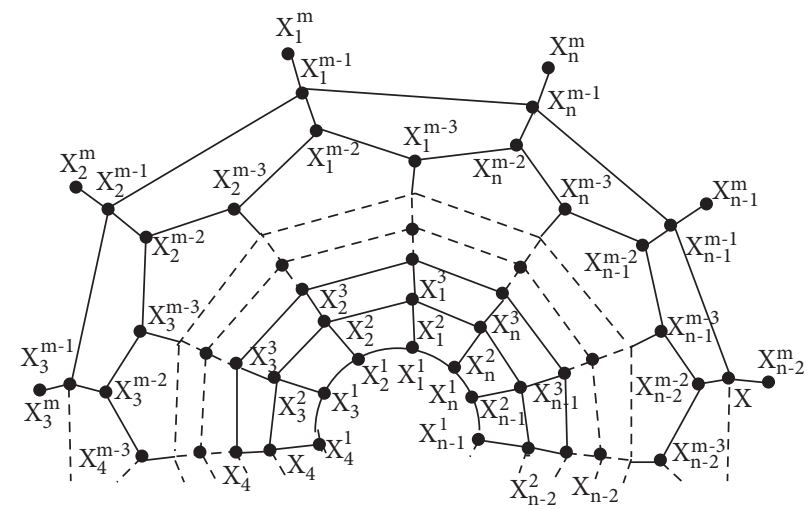

Figure 3: The generalized convex polytope graph $U_{n, m}^{p}$.

For $2 \leq j \leq m-3$,

$$
r\left(X_{s}^{j} \mid Z\right)=(j-1, j-1, j-1)+r\left(X_{s}^{1} \mid Z\right) .
$$

Representation given to the $X_{i}^{m-2}$ cycle is

$$
r\left(X_{s}^{m-2} \mid Z\right)= \begin{cases}\left(3,3, \alpha^{\prime}+2\right), & s=1 \\ \left(s+2, s+1, \alpha^{\prime}-s+3\right), & \alpha^{\prime} \geq s \geq 2, \\ \left(\alpha^{\prime}+2, \alpha^{\prime}+2,3\right), & s=\alpha^{\prime}+1 \\ \left(2 \alpha^{\prime}-s+3,2 \alpha^{\prime}-s+4, s-\alpha^{\prime}+2\right), & 2 \alpha^{\prime} \geq s \geq \alpha^{\prime}+2 .\end{cases}
$$

Representation of the vertices of the outer cycle is

$$
r\left(X_{s}^{m-1} \mid Z\right)=(1,1,1)+r\left(X_{s}^{m-2} \mid Z\right), \quad 1 \leq s \leq n .
$$

Representation of pendent vertices is

$$
r\left(X_{s}^{m} \mid Z\right)=(2,2,2)+r\left(X_{s}^{m-2} \mid Z\right), \quad 1 \leq s \leq n .
$$

It shows that $Z$ is a resolving set for GCP $U_{n, m}^{p}$ implying that $\operatorname{dim}\left(U_{n, m}^{p}\right)=3$.

Case (ii): when $n$ is an odd integer. Let $n=2 \alpha^{\prime}+1$ for $\alpha^{\prime} \geq 3$; let $Z=\left\{X_{1}^{1}, X_{2}^{1}, X_{l+1}^{1}\right\}$ be an ordered subset of the vertices of the GCP. To show that $Z$ is a locating set for $U_{n, m}^{p}$, consider the codes' formulation of the vertices of the GCP with respect to $Z$ as

$$
r\left(X_{1}^{1} \mid Z\right)= \begin{cases}\left(s-1, s-2, \alpha^{\prime}-s+1\right), & \alpha^{\prime} \geq s \geq 3, \\ \left(\alpha^{\prime}, \alpha^{\prime}, 1\right), & s=\alpha^{\prime}+2, \\ \left(2 \alpha^{\prime}-s+2,2 \alpha^{\prime}-s+3, s-\alpha^{\prime}-1\right), & 2 \alpha^{\prime}+1 \geq s \geq \alpha^{\prime}+3\end{cases}
$$


For $2 \leq j \leq m-2$,

$$
r\left(X_{s}^{j} \mid Z\right)=(j-1, j-1, j-1)+r\left(X_{s}^{1}\right) .
$$

Representation given to the vertices of the interior cycle

$$
r\left(X_{s}^{m-2} \mid Z\right)= \begin{cases}\left(3,3, \alpha^{\prime}+2\right), & s=1, \\ \left(s+2, s+1, \alpha^{\prime}-s+3\right), & \alpha^{\prime} \geq s \geq 2, \\ \left(\alpha^{\prime}+3, \alpha^{\prime}+2,3\right), & s=\alpha^{\prime}+1, \\ \left(\alpha^{\prime}+2, \alpha^{\prime}+1,4\right), & s=\alpha^{\prime}+2, \\ \left(\alpha^{\prime}+1, \alpha^{\prime}, 5\right), & s=\alpha^{\prime}+3, \\ \left(2 \alpha^{\prime}-s+4,2 \alpha^{\prime}-s+5, s-\alpha^{\prime}+2\right), & \\ 2 \alpha^{\prime}+1 \geq s \geq \alpha^{\prime}+3 . & \end{cases}
$$

Representation given to the nodes of the outer cycle is

$$
r\left(X_{s}^{m-1} \mid Z\right)=(1,1,1)+r\left(X_{s}^{m-2} \mid Z\right), \quad 1 \leq s \leq n .
$$

Also, representation given to the nodes hanging is

$$
\left(X_{s}^{m} \mid Z\right)=(2,2,2)+r\left(X_{s}^{m-2} \mid Z\right), \quad 1 \leq s \leq n .
$$

It gives us that $Z$ is a resolving set for GCP $U_{n, m}^{p}$, implying that the metric dimension of GCP $U_{n, m}^{p}$ is 3 .

\section{Concluding Remarks}

In this paper, we focus to study those graphs obtained from convex polytopes and examine that generalized convex polytopes (GCPs) also belong to the family of constant metric dimension such as their parent graphs $S_{n}, T_{n}$, and $U_{n}$.

\section{Data Availability}

The data used to support the findings of this study are cited at relevant places within the text as references.

\section{Conflicts of Interest}

The authors declare that they have no conflicts of interest.

\section{Authors' Contributions}

All authors contributed equally to this work.

\section{References}

[1] J.-B. Liu, M. F. Nadeem, H. M. A. Siddiqui, and W. Nazir, "Computing metric dimension of certain families of toeplitz graphs," IEEE Access, vol. 7, pp. 126734-126741, 2019.

[2] I. Muhammad, A. Q. Baig, and A. Ali, "Families of plane graphs with constant metric dimension," Utilitas Mathematica, vol. 88, pp. 43-53, 2012.

[3] C. Gary, L. Eroh, M. A. Johnson, and O. R. Oellermann, "Resolvability in graphs and the metric dimension of a graph," Discrete Applied Mathematics, vol. 105, no. 1-3, pp. 99-113, 2000.
[4] I. Tomescu, J. Imran, and S. Imran, "On the partition and connected partition dimension of Wheels," Ars Combinatoria, vol. 84, pp. 311-318, 2007.

[5] B. Peter, C. Gary, C. Poisson, and P. Zhang, "On k-dimensional graphs and their bases," Periodica Mathematica Hungarica, vol. 46, no. 1, pp. 9-15, 2003.

[6] J. Cáceres, C. Hernando, M. Mora et al., "On the metric dimension of some families of graphs," Electronic Notes in Discrete Mathematics, vol. 22, no. 2, pp. 129-133, 2005.

[7] I. Tomescu and J. Imran, "On the metric dimension of the Jahangir graph," Bulletin mathématique de la Société des Sciences Mathématiques de Roumanie, vol. 7, pp. 371-376, 2007.

[8] I. Javaid, On metric and partition dimension of some wheel related graphs, $\mathrm{PhD}$ Thesis, GC University Lahore, Lahore, Pakistan, 2007.

[9] I. Javaid, M. T. Rahim, and K. Ali, "Families of regular graphs with constant metric dimension," Utilitas Mathematica, vol. 75, pp. 21-33, 2008.

[10] S. Khuller, B. Raghavachari, and A. Rosenfeld, "Landmarks in graphs," Discrete Applied Mathematics, vol. 70, no. 3, pp. 217-229, 1996.

[11] B. Grünbaum and G. C. Shephard, "Convex polytopes," Bulletin of the London Mathematical Society, vol. 1, no. 3, pp. 257-300, 1969.

[12] M. Imran, S. A. Ul Haq Bokhary, and A. Q. Baig, "On the metric dimension of convex polytopes," AKCE International Journal of Graphs and Combinatorics, vol. 10, no. 3, pp. 295-307, 2013.

[13] C. Gary, E. Salehi, and P. Zhang, "The partition dimension of a graph," Aequationes Mathematicae, vol. 59, pp. 45-54, 2000.

[14] I. Tomescu, "On the ratio between partition dimension and metric dimension of a connected graph," Analele Universitatii Bucuresti. Matematica-Informatica, vol. 55, pp. 3-10, 2006.

[15] Z. Shao, S. M. Sheikholeslami, Pu Wu, and J.-B. Liu, "The metric dimension of some generalized Petersen graphs," Discrete Dynamics in Nature and Society, vol. 8, pp. 19-29, 2018.

[16] M. Imran, S. A. Ul Haq Bokhary, and A. Q. Baig, "On families of convex polytopes with constant metric dimension," Computers and Mathematics with Applications, vol. 60, no. 9, pp. 2629-2638, 2010. 